\title{
Assessment of Ultrasound Velocity Application for Chemical Process Monitoring
}

\author{
Linas Svilainis ${ }^{1}$, Alberto Rodriguez ${ }^{2}$, Dobilas Liaukonis ${ }^{1}$, Andrius Chaziachmetovas ${ }^{1}$ \\ ${ }^{I}$ Department of Electronics Engineering, Kaunas University of Technology, \\ Studentu St. 50-340, LT-51368 Kaunas, Lithuania \\ ${ }^{2}$ Department of Communications Engineering, Universidad Miguel Hernandez, \\ Avda. Universidad S/N, 03203 Elche, Spain \\ linas.svilainis@ktu.lt
}

\begin{abstract}
Study on the accuracy of the ultrasound velocity application for chemical process monitoring is presented. Cure degree of epoxy resins was monitored in order to establish the percentage of the final cure based on ultrasound velocity of longitudinal waves. Time of flight estimations by correlation processing were used to estimate the velocity. Sources of error in the velocity measurement and their influence in the sensitivity and accuracy of the results have been studied. In order to improve the accuracy and reliability of the measurements, the use of Spread Spectrum instead of conventional excitation signals have been evaluated. Experimental results indicate that random errors of propagation time estimation are lower than velocity fluctuation over the chemical process. Spread spectrum signals provide more reliable measurements.
\end{abstract}

Index Terms-Ultrasonic variables measurement, spread spectrum signals, time of arrival estimation, estimation error, measurement uncertainty, chemical process monitoring.

\section{INTRODUCTION}

Composite materials are one of the most used materials in the industry nowadays, due to their mechanical properties, combining strength and lightness and because of their reduced price and ease of manufacturing. Most of those materials are manufactured using polymers like epoxy resin matrices as basis adhesive for different reinforcement fibers, such as glass-fiber, carbon-fiber, aramid, etc. The mechanical properties of the final product are strongly dependent on the curing process of the epoxy resins and the cross-linked reinforcement structure, obtained by reaction of the linear epoxy resin with suitable curatives. For these reasons, the estimation of the cure level is crucial during the product development stage to ensure the sustainability of the production quality [1], [2]. Furthermore, monitoring the cure process is required to predict the mould removal time [3], [4].

For the aforementioned purposes, ultrasound has been widely used for components quality inspection [5], [6], mainly because ultrasonic sensors offer instantaneous evaluation of the status and do not require a direct contact to the specimen under analysis [7], [8]. Usually, the ultrasound attenuation [1], [3] and/or the propagation velocity [2], [7] are measured for curing state estimation. The techniques based on the measurement of the velocity involve precise timing of the signal propagation; therefore wide signal bandwidth and high signal-to-noise ratio (SNR) are required. Conventional signals do not offer both parameters: pulse signals have wide bandwidth but low SNR; long continuous wave $(\mathrm{CW})$ tonebursts has high SNR but narrow bandwidth and also suffer from abrupt errors in peak estimation, resulting in a low temporal resolution.

The aim of this investigation was to assess the reliability of the monitoring process based on ultrasound velocity measurements of the chemical curing process. For this purpose, we introduce the use of spread spectrum (SS) signals instead of conventional pulse or CW signals. SS signals offer the additional advantage of increased energy and wide bandwidth. Binary spread spectrum signals are of particular interest since are easy to generate and offer significant cost besides allowing the reduction of the size of the test equipment.

In addition, we analyse the different sources of error in the velocity measurements and their influence in the accuracy of the estimates.

\section{VElocity Estimation Procedure}

The speed of ultrasound is related to the elastic modulus and density of the material [1], [3], [7]. Due to the fact that changes in resin elasticity will affect the velocity of the ultrasound propagation, it is expected that ultrasound velocity will reflect the state of the chemical process. In order to calculate the ultrasound velocity, $c_{\text {epox }}$, two values are needed: the time of flight of the ultrasonic signal in the material, $T o F$, and the distance of propagation, $L_{\mathrm{epox}}$

$$
c_{\text {epox }}=\frac{L_{\text {epox }}}{T o F} \text {. }
$$

Estimation of the time-of-flight (ToF) was based on the cross-correlation maximum criteria [9], [10], where global signal properties are exploited. Discrete version of the crosscorrelation function $x_{R T}[m]$ can be calculated as

$$
x_{R T}[m]=\sum_{k=0}^{M-1} s_{R}[k] s_{T}[m-k],
$$


where $s_{T}[k]$ and $s_{R}[k]$ are the discrete versions of the transmitted and corresponding received ultrasonic signals and $M$ the record length. Because cross-correlation establishes the maximum likelihood between signals, discrete time instant $m$ that maximizes $x_{R T}[m]$ will provide the delay between them, and therefore the desired ToF

$$
T o F_{D C}=\arg \left[\max \left(x_{R T}[m]\right)\right] .
$$

Unfortunately, due to sampling only rough ToF estimate can be obtained. Sub index DC of ToF in (3) indicates the discrete nature of the estimate. In order to obtain the subsample values of the ToF, interpolation around this maximum location is carried out. As indicated in [10] and [11], best results are obtained for cosine interpolation

$$
\Delta T o F=\frac{\theta}{f_{s} \omega_{0}},
$$

where $\Delta T o F$ is the interpolated subsample ToF, $f_{\mathrm{s}}$ is the sampling frequency and $\omega_{0}$ the center angular frequency of the signal

$$
\omega_{0}=\arccos \left(\frac{x_{R T}[m-1]+x_{R T}[m+1]}{2 x_{R T}[m]}\right),
$$

and $\theta$ is the phase

$$
\theta=\arctan \left(\frac{x_{R T}[m-1]-x_{R T}[m+1]}{2 x_{R T}[m] \sin \left(\omega_{0}\right)}\right) .
$$

Full, subsample interpolated $T o F$ value is the sum of the two estimates

$$
T o F=T o F_{D C}+\Delta T o F
$$

An additional measurement should be carried out where transducers were placed against each other with no gap in order to compensate the ToF bias error caused by signal propagation in electronics, cabling and ultrasonic transducers. ToF obtained under these conditions (labeled as $\left.T o F_{0}\right)$ will be subtracted from ToF values to obtain the final estimation

$$
\text { ToF } F_{\text {corr }}=\text { ToF }- \text { ToF }_{0} \text {. }
$$

This $T o F_{\text {corr }}$ is the value used in (1) to obtain non-biased estimate of the ultrasound velocity.

\section{RANDOM ERROR ANALYSIS AND ACCURACY ESTIMATION}

Statistical sensitivity of the velocity estimation due to random errors in measurements can be calculated as the mean square of the sensitivities of the parameters involved in its calculation [12]. Thus, according to (1), sensitivity of the velocity estimation $\sigma(c)$ will be the mean square of the sensitivities due to random errors in the measurement of the ToF, $\sigma\left(c_{T o F}\right)$, and the thickness, $\sigma\left(c_{L}\right)$, respectively

$$
\sigma(c)=\sqrt{\sigma\left(c_{L}\right)^{2}+\sigma\left(c_{T o F}\right)^{2}} .
$$

Sensitivity due to random errors in ToF measurement can be calculated taking partial derivative of (1) over ToF as

$$
\sigma\left(c_{T o F}\right)=\frac{\partial c}{\partial T o F} \sigma(T o F)=-\frac{L}{T o F^{2}} \sigma(T o F),
$$

where random errors in the ToF estimation are defined by Cramer-Rao lower error bound [10]

$$
\sigma(T o F) \geq \frac{1}{2 \pi F_{e} \sqrt{S N R}}
$$

The signal to noise ratio $(S N R)$ is in this case expressed as the energy to noise power spectral density ratio

$$
S N R=\frac{2 E}{N_{0}},
$$

where the energy $E$ is calculated as

$$
E=\int_{-\infty}^{\infty}|s(t)|^{2} d t
$$

and the noise power spectral density $N_{0}$ is estimated using the DFT as

$$
N_{0}=\frac{2 \sum_{n=1}^{N_{\text {avg }}}\left|\sum_{m=0}^{M-1} s_{R}[m] e^{-i \frac{2 \pi}{N} m k}\right|^{2}}{f_{s} N_{\text {avg }} M},
$$

where $N_{\text {avg }}$ is the number of averaging cycles taken to obtain better noise spectrum statistics and $M$ the record length. It have to be noted that neither noise nor energy calculation involve the impedance since only the ratio of the last is needed in (12).

The effective signal bandwidth $F_{\mathrm{e}}$ can be calculated as the squared sum of the envelope bandwidth $\beta$ and the centre frequency $f_{0}$

$$
F_{e}^{2}=\beta^{2}+f_{0}^{2}
$$

where the envelope bandwidth $\beta$ and the centre frequency $f_{0}$ are calculated respectively as

$$
f_{0}=\frac{\int_{-\infty}^{\infty} f|S(f)|^{2} d f}{E}
$$

and

$$
\beta^{2}=\frac{\int_{-\infty}^{\infty}\left(f-f_{0}\right)^{2}|S(f)|^{2} d f}{E},
$$


On the other hand, sensitivity due to random errors in thickness estimation, $\sigma\left(c_{L}\right)$, can be established in the same way, taking partial derivative of (1) over measurement base $L$

$$
\sigma\left(c_{L}\right)=\frac{\partial c}{\partial L} \sigma(L)=\frac{1}{T o F} \sigma(L) .
$$

The estimation of the standard deviation of the thickness measurement, $\sigma(L)$, depends on the measurement procedure. In this case, measurements are carried out by scanner and additionally confirmed on the cured sample by taking micrometer readings. In this experiment both devices have $10 \mu \mathrm{m}$ resolution, $\Delta L$. Thus, the standard deviation of the measurement base estimation using scanner or micrometer is

$$
\sigma(L)=\frac{d L}{\sqrt{12}} .
$$

Finally, regarding the accuracy, subsample estimation of ToF also involves the interpolation bias error $\varepsilon(T o F)$ [10], [11]. For cosine interpolation, the maximum value of the interpolation bias error can be found [10] as

$$
\max [\varepsilon(T o F)]=\sqrt{\pi} \frac{\beta^{2}}{f_{s}^{3}} .
$$

\section{EXPERIMENTAL SETUP}

The experiment setup is shown in Fig. 1. The test chamber was manufactured to monitor the ultrasound velocity by direct contact of the ultrasonic transducers with the resin mixture.

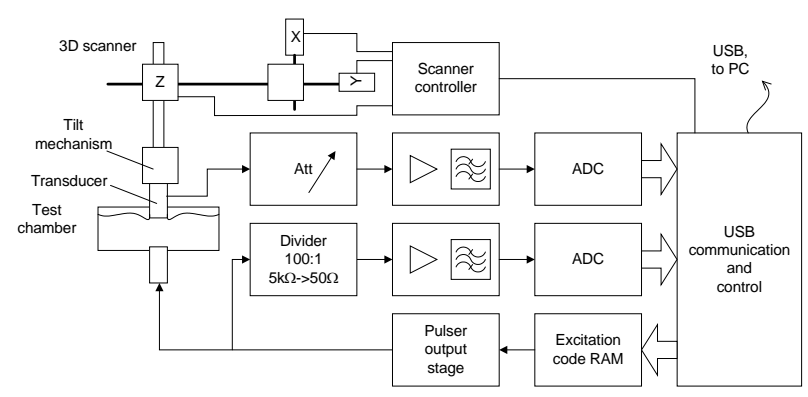

Fig. 1. Experimental setup structure used for data collection.

A $2 \mathrm{MHz}$ centre frequency ultrasonic transducer TF2C6N from Dopler Inc. was used for probing signal transmission. It was mounted at the bottom of the chamber. The face of the transducer was protected by a $40 \mu \mathrm{m}$ thin sticky polypropylene tape and coated by silicone grease. Another transducer (5 MHz centre frequency C543-SM from Olympus) was positioned at the top using a 3D scanner [8].

Distance between transducers $(5 \mathrm{~mm})$ was controlled using the scanner $\mathrm{Z}$ axis ( $5 \mu \mathrm{m}$ resolution). Before starting the experiment, the upper transducer was lowered and adjusted by $x-y$ scanning axes tilt mechanism $(10 \mu \mathrm{m}$ resolution) to come in full contact with the lower transducer face (confirmation by ultrasound penetration and signal position in time). This position was considered as reference (distance between transducers $0 \mathrm{~mm}$ ). After raising the transducer and pouring the mixed resin into the chamber, the upper transducer was lowered again to make $5 \mathrm{~mm}$ distance to the lower transducer face.

Acquisition of the signals was carried out using universal ultrasonic data acquisition system [13]. Bipolar pulser was used for excitation. Excitation signals were routed for registration using 100:1 divider, with input impedance of $5 \mathrm{k} \Omega$. Signal from the receiving transducer was directly fed to a programmable attenuator. The output of the divider and programmable attenuator were registered with two 10 bit $100 \mathrm{Ms} / \mathrm{s}$ ADC channels. Control and data communication to host PC were carried out using high speed USB interface.

It must be noted that the transmitting and receiving transducers have a different centre frequency of operation. Such arrangement of the transducers results in maximally flat transmission response over a wider frequency domain. Transmission response of such transducers arrangement was measured using $0.5 \mathrm{MHz}$ to $5 \mathrm{MHz} 3 \mu \mathrm{s}$ long linear frequency modulation chirp to confirm the shape of the frequency domain response. Sampled signals $(M=32 \mathrm{k}$ samples) registered on transmitting transducer, $s_{T}[m]$, and the signal on receiving transducer, $s_{R}[m]$, were registered while transducers were arranged in direct contact. Then signals were transformed into frequency domain using Discrete Fourier Transform (DFT) and magnitudes divided, producing the following transmission frequency response

$$
T_{k}=\frac{\left|\sum_{m=0}^{M-1} s_{R}[m] e^{-i \frac{2 \pi}{N} m k}\right|}{\left|\sum_{m=0}^{M-1} s_{T}[m] e^{-i \frac{2 \pi}{N} m k}\right|} .
$$

Transmission AC response measured in such way is presented in Fig. 2.

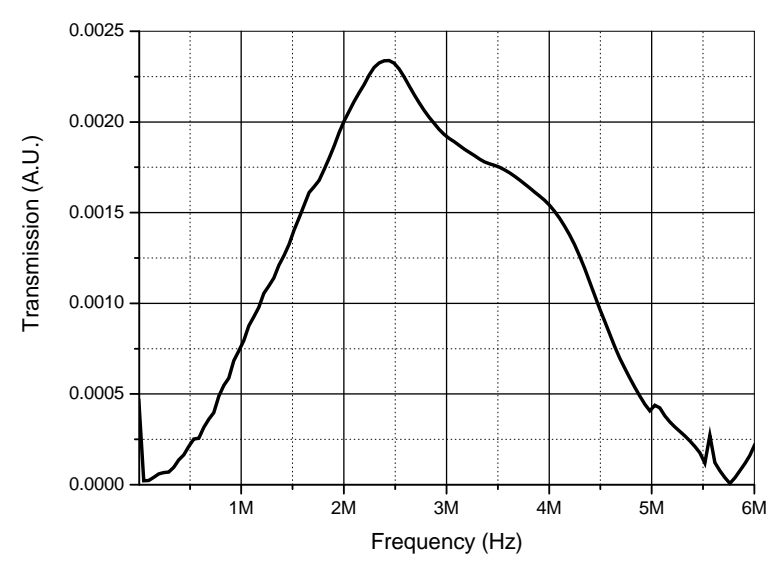

Fig. 2. Transmission $\mathrm{AC}$ response of $2 \mathrm{MHz}$ and $5 \mathrm{MHz}$ transducer pair.

Dual component epoxy system Araldite was analysed in the experiments. It contains hardener XB3404-1 and base resin Araldite LY1564. Components were mixed in 100:36 proportions by weight using digital scales DI003 (0.1 g resolution). This type of epoxy requires curing at elevated temperatures, thus curing was deliberately carried out at room temperature $\left(20^{\circ} \mathrm{C}\right)$ to avoid the complete cure. The chemical linkage process was monitored for $138 \mathrm{~h}$. A sample was additionally measured after 10 days [14] and finally was post-cured at elevated temperature $\left(60^{\circ} \mathrm{C}\right)$, 
measuring the ultrasound velocity again.

Four signals were chosen to perform the comparison between the accuracy obtained with conventional (pulse or toneburst) signals and proposed Spread Spectrum signals: rectangular $500 \mathrm{~ns}$ duration (optimal for $2 \mathrm{MHz}$ ) pulse signal (Fig. 3); CW $2 \mathrm{MHz}$ toneburst (Fig. 4) and two chirp signals: $0.5 \mathrm{MHz}$ to $5 \mathrm{MHz}$ (best bandwidth, Fig. 5) and 0.7 MHz to 3.5 MHz (best SNR, Fig. 6).

All excitation signals were rectangular pulses or pulse trains with bipolar $\pm 200 \mathrm{~V}$ amplitude. All (except pulse) had $3 \mu$ s duration so initial energy was the same. Acquisition sampling frequency was set to $100 \mathrm{MHz}$ for the whole experiment.

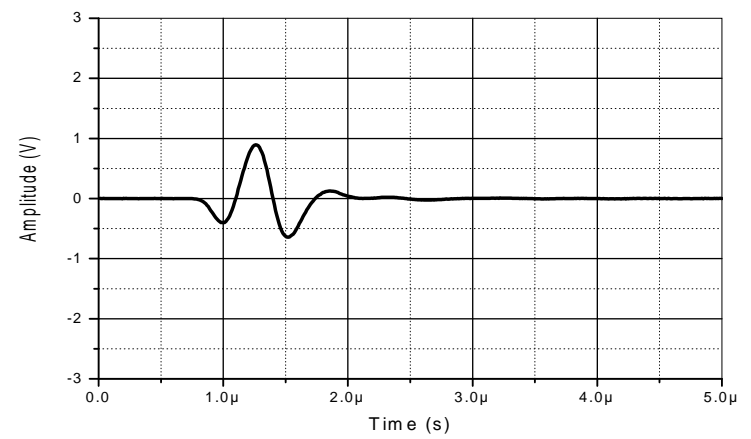

Fig. 3. Received signal when rectangular pulse is used for excitation.

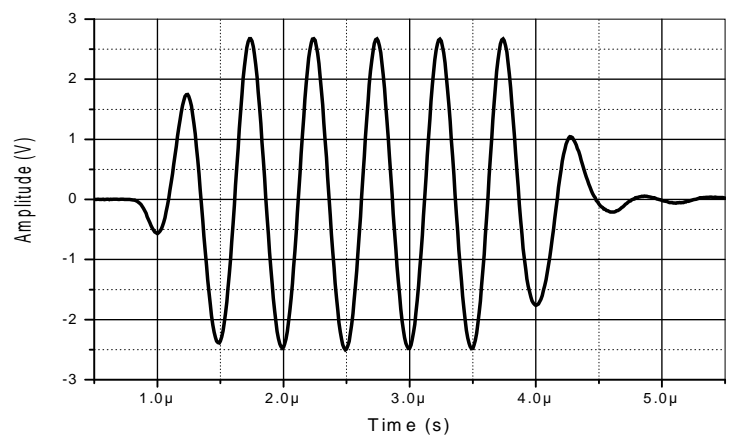

Fig. 4. Received signal in case of rectangular $\mathrm{CW}$ toneburst excitation.

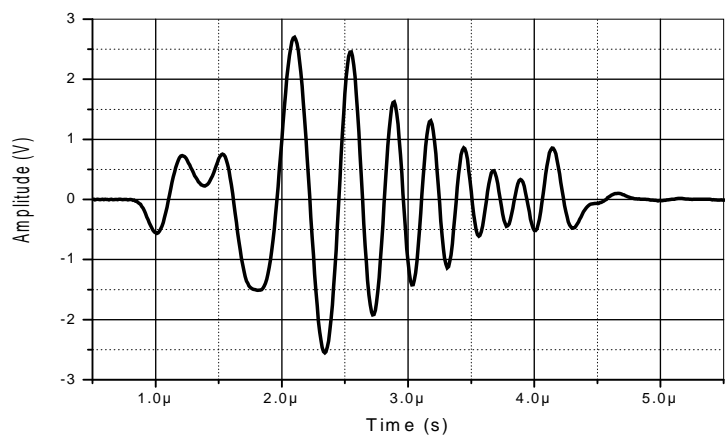

Fig. 5. Received signal for rectangular (0.5-5) MHz chirp excitation.

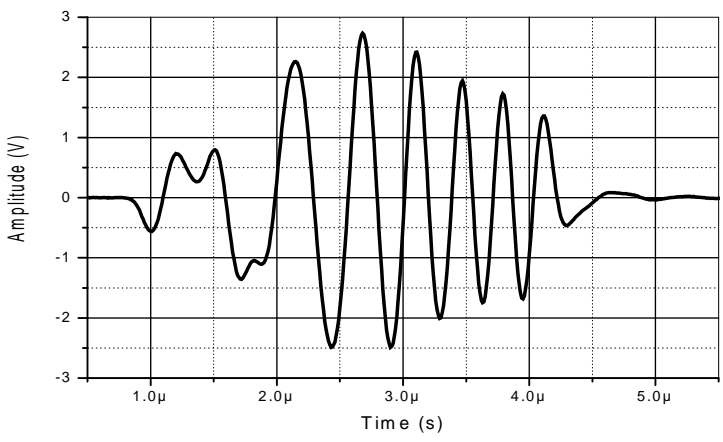

Fig. 6. Received signal for rectangular (1-3.5) MHz chirp excitation.

\section{RESULTS}

Acquired data was used to calculate the ultrasound velocity over entire experiment cycle using (1)-(8). Estimated velocity values for the whole period of the experiment over curing stages are shown in Fig. 7.

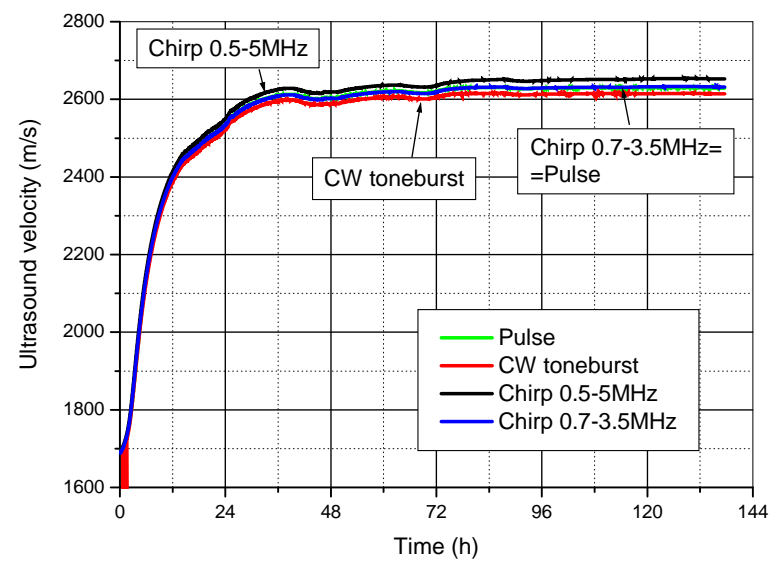

Fig. 7. Ultrasound velocity variation during epoxy curing process.

Three states of the chemical linkage process can be separated. Initially, resin is liquid so the velocity $(1600 \mathrm{~m} / \mathrm{s}-$ $1750 \mathrm{~m} / \mathrm{s}$ ) is close to that of water. Gelation phase can be identified by rapid change of velocity $(1800 \mathrm{~m} / \mathrm{s}$ to $2500 \mathrm{~m} / \mathrm{s}$ ). Attenuation in this phase was high; therefore a programmable attenuator was used to automatically adjust the level of the signal in order to keep the SNR high. Solidification phase has slow convergence of the ultrasound velocity to $2800 \mathrm{~m} / \mathrm{s}$. Attenuation at this phase drops down, so the programmable attenuator was used again to match the signal dynamic range to the ADC input.

Difference between fully cured (elevated temperature for $24 \mathrm{~h}$ ) and main experiment velocities can be related to the degree of cure (Fig. 8). Assuming that final cure (100\%) can be assigned to velocity $c_{100}=2720 \mathrm{~m} / \mathrm{s}$ and taking the velocity $c_{0}=1600 \mathrm{~m} / \mathrm{s}$ as uncured condition (base resin without hardener, $0 \%$ ), the degree of cure is calculated as

$$
T o F_{\text {corr }}=\frac{c_{\text {epox }}-c_{0}}{c_{100}-c_{0}} \times 100 \%
$$

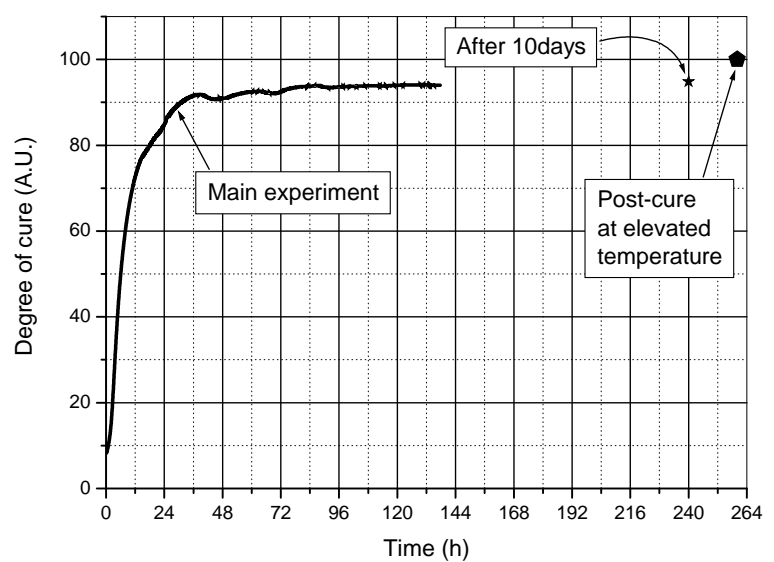

Fig. 8. Degree of cure estimated from ultrasound velocity. Results for $0.5 \mathrm{MHz}$ to $5 \mathrm{MHz}$ chirp.

It can be seen in Fig. 8 that the estimation of the degree of cure is significantly affected by accuracy of the velocity estimation. The estimate of the lower error bound for ToF 
random error obtained using (11) is shown in Fig. 9.

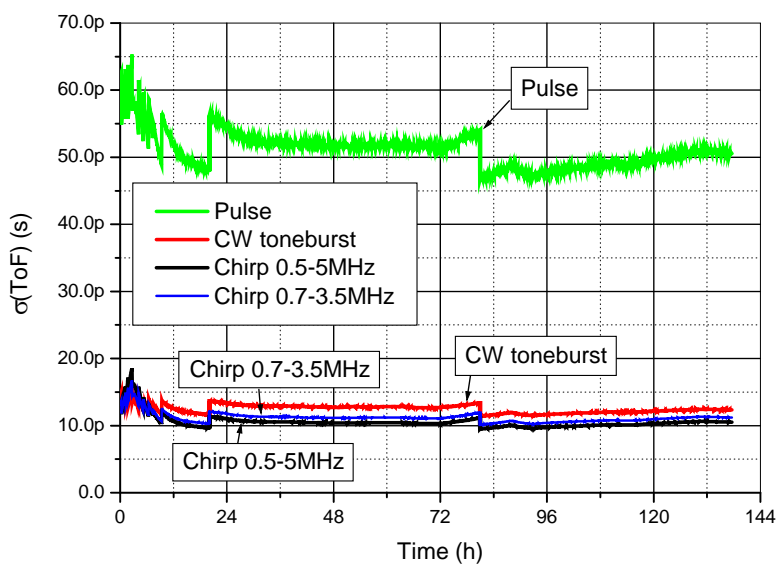

Fig. 9. Theoretical random errors of the ToF estimation obtained from measured system parameters.

Taking the envelope bandwidth $\beta=1.8 \mathrm{MHz}$ and sampling frequency $f_{\mathrm{s}}=100 \mathrm{MHz}$, the resulting maximum value of the interpolation error $\varepsilon\left(T o F_{\mathrm{ss}}\right)$ according to (20) is 5.5 ps. Comparing this value with the results shown in Fig. 9 (10 ps minimum) it can be concluded that interpolation errors can be neglected.

Figure 9 also shows that the lowest random errors are expected for wideband chirp ( $0.5 \mathrm{MHz}$ to $5 \mathrm{MHz}$ ), while worst performance is expected for the pulse signal (because of the lower energy). On the other hand, a significant disadvantage can be seen for $\mathrm{CW}$ toneburst signal: there are abrupt ToF estimation errors, which are caused by peak location on neighbouring CW period.

Random errors of the time scale can be neglected taking into account that reference clock oscillator (ASEMPC) have 3 ps jitter. Time scale bias errors are defined by initial frequency stability of the oscillator $(10 \mathrm{ppm})$, oscillator ageing ( $5 \mathrm{ppm} / \mathrm{year}$ ) and the ToF being measured. Assuming $15 \mathrm{ps}$ as standard deviation for the time scale bias error (1 year of exploitation) and taking ToF values from the experiment, the expected velocity estimation bias errors are shown in Fig. 10.

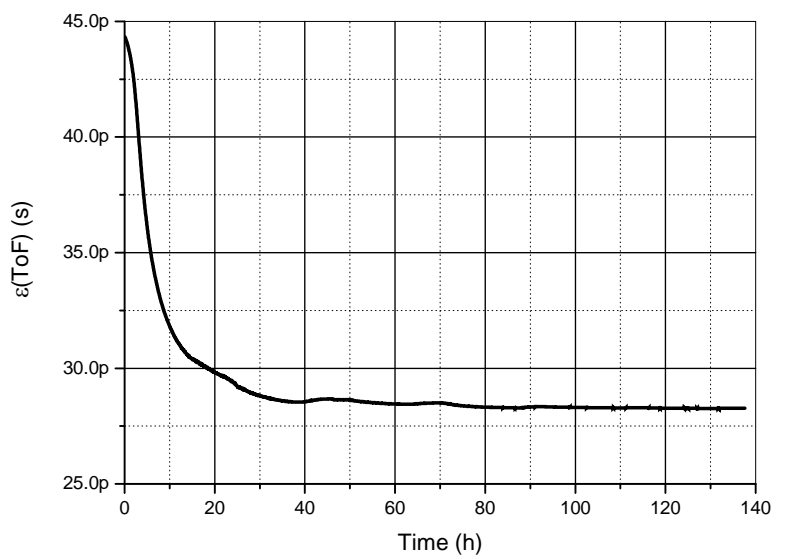

Fig. 10. Theoretical random errors of the ToF estimation caused by time scale bias.

Figure 11 shows the results of the total expected errors of the velocity estimation which are influenced by the ToF errors according to (10). It can be seen that expected errors for SS signals and CW burst are almost six times lower than that for pulse. Results can be predicted since energy of the pulse is low: then ToF estimation errors are higher, so are the ultrasound velocity estimation errors.

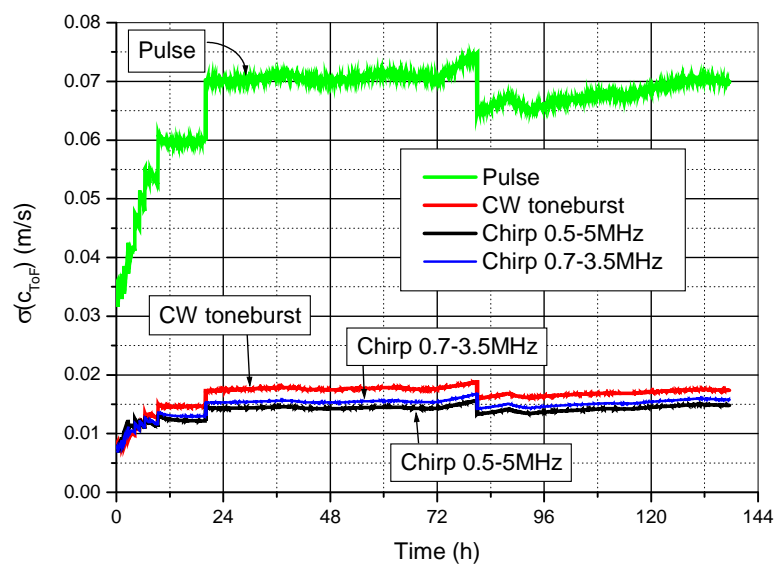

Fig. 11. Theoretical velocity estimation errors caused by the ToF estimation random errors.

Another source of the velocity estimation errors is related to the estimation of the propagation path length. Theory on this error source evaluation has been presented in Section III. Figure 12 shows the results of the sensitivity due to the estimation of the epoxy thickness according to (19).

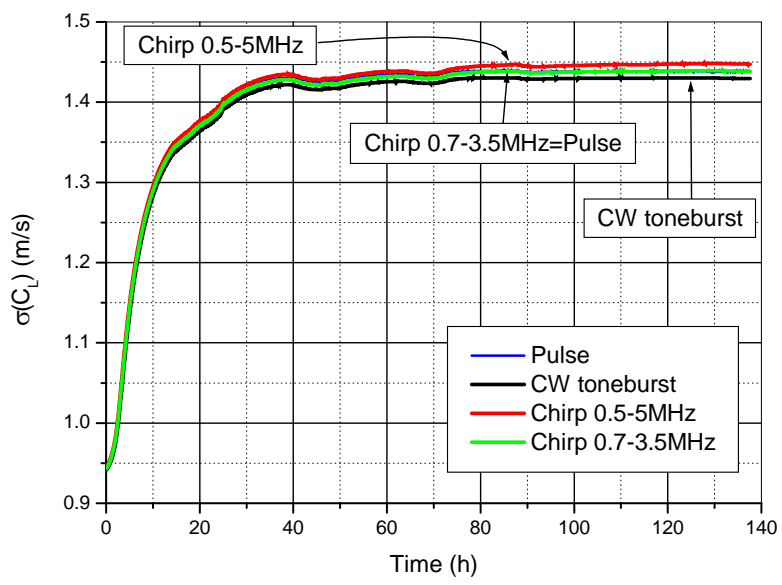

Fig. 12. Theoretical velocity estimation errors caused by the thickness estimation errors.

The total error for the velocity estimation is obtained combining the previous results according to (9), as shown in Fig. 13, where it can be seen that thickness estimation errors prevail. Therefore, difference between the accuracy obtained by different signals is small.

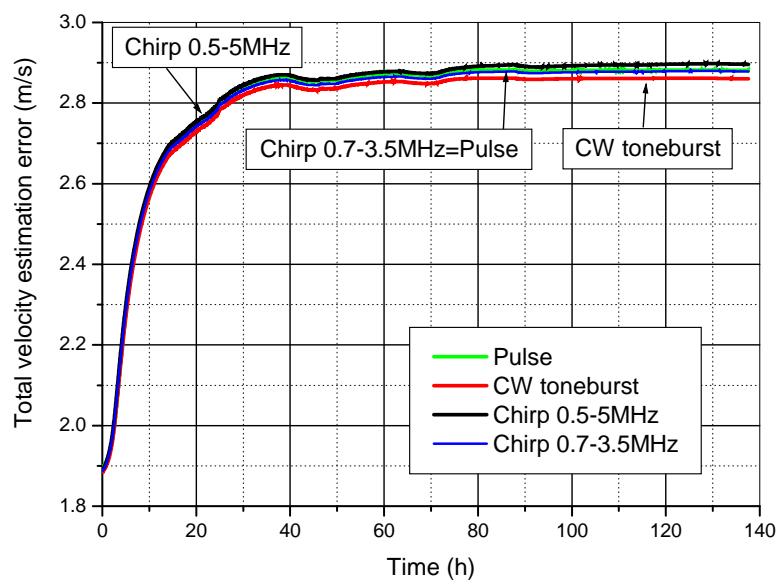

Fig. 13. Theoretical total uncertainty of the velocity estimation. 
Finally, Fig. 14 shows the velocity values for the end of curing with its confidence interval. It can be seen that errors obtained by theoretical analysis, using measured system parameters, are low enough to give the confidence in chemical process monitoring.

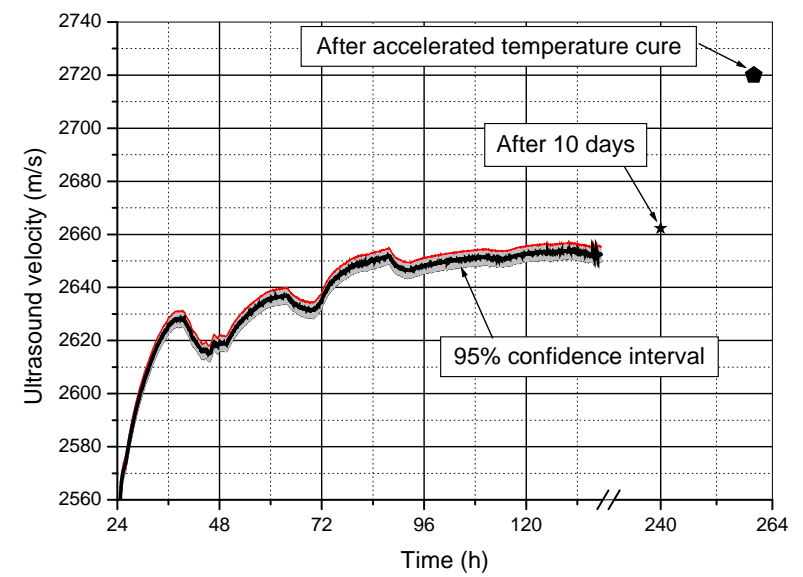

Fig. 14. Measured velocity for curing end with confidence interval shown.

Value of this research lies in the evaluation of the errors and comparison between different signal types. Future study should account other sources of errors (such as transducers attachment or temperature) so these are handled properly to reliably indicate the process stage.

\section{CONCLUSIONS}

In this study, the assessment of the chemical process monitoring using the ultrasound velocity has been carried out. The results indicate that time of flight estimation errors are strongly influenced by the signal energy. The lowest errors are obtained for wideband chirp, so its use is recommended to improve the accuracy and reliability of the monitoring process. The worst performance, due to low energy, is for the pulse signal. CW toneburst has a significant disadvantage: abrupt ToF estimation errors occur are caused by peak location on neighbouring $\mathrm{CW}$ period even at significant SNR level.

On the other hand, the difference in accuracy between the signals is small because thickness estimation errors prevail in the final error budget. This study indicates also that the uncertainty in the velocity estimation due to the parameters of the measurement system is low enough to ensure the confidence in the chemical process monitoring.

Further study is needed to assure that other sources of errors are handled properly to reliably predict the end of chemical curing process, especially those concerning the transducers attachment and the temperature monitoring.

\section{REFERENCES}

[1] W. Stark, H. Goering, U. Michel, H. Bayerl, "Online monitoring of thermoset postcuring by dynamic mechanical thermal analysis", in Proc. DMTA. Polym. Test., vol. 28, pp. 561-566, 2009. [Online]. Available: http://dx.doi.org/10.1016/j.polymertesting.2009.02.005

[2] F. Lionetto, A. Maffezzoli, "Monitoring the cure state of thermosetting resins by ultrasound", Materials, vol. 6, pp. 3783-3804, 2013. [Online] Available: http://dx.doi.org/10.3390/ma6093783

[3] A. G. Mamalis, N. G. Pantelelis, K. N. Spentzas, "Process monitoring and cure control applied in RTM production of epoxy/carbon fibre parts", in Proc. TMS-ABM International Materials Congress, Rio de Janeiro, Brazil, 2010, pp. 4568-4576.

[4] N. Liebers, F. Raddatz, F. Schadow, "Effective and flexible ultrasound sensors for cure monitoring for industrial composite production", Deutsche Gesellschaft für Luft- und Raumfahrt Lilienthal-Oberth e.V., pp. 1-6, 2012.

[5] P. J. Schubel, R. J. Crossley, E. K. G. Boateng, J. R. Hutchinson, "Review of structural health and cure monitoring techniques for large wind turbine blades", Renewable Energy, vol. 51, pp. 113-123, 2013. [Online] Available: http://dx.doi.org/10.1016/j.renene.2012.08.072

[6] V. Samaitis, L. Mazeika, "Investigation of diffuse LambWave Sensitivity to the Through-Thickness Notch in Structural Health monitoring of composite objects", Elektronika Ir Elektrotechnika, vol. 20, no. 3, pp. 48-51, 2014. [Online] Available: http://dx.doi.org/10.5755/j01.eee.20.3.6690

[7] Z Luo, H. Zhu, J. Zhao, "In Situ monitoring of epoxy resin curing process using ultrasonic technique", Experimental Techniques, vol. 36, no. 2, pp. 6-11, 2012. [Online] Available: http://onlinelibrary.wiley.com/doi/10.1111/j.17471567.2011.00741.x/full

[8] L. Svilainis, V. Dumbrava, S. Kitov, A. Aleksandrovas, P. Tervydis, D. Liaukonis, "Electronics for ultrasonic imaging system", Elektronika Ir Elektrotechnika, vol. 20, no. 7, pp. 51-56, 2014. [Online] Available: http://dx.doi.org/10.5755/j01.eee.20.7.8024

[9] M. R. Hoseini, X. Wang, M. J. Zuo, "Estimating ultrasonic time of flight using envelope and quasi maximum likelihood method for damage detection and assessment", Measurement, vol. 45, pp. 20722080, 2012. [Online] Available: http://dx.doi.org/10.1016/j.measurement.2012.05.008

[10] L. Svilainis, K. Lukoseviciute, V. Dumbrava, A. Chaziachmetovas, "Subsample interpolation bias error in time of flight estimation by direct correlation in digital domain", Measurement, vol. 46, pp. 39503958, 2013. [Online] Available: http://dx.doi.org/10.1016/j.measurement.2013.07.038

[11] I. Cespedes, Y. Huang, J. Ophir, S. Spratt, "Methods for estimation of subsample time delays of digitized echo signals", Ultrasonic Imaging, vol. 17, pp. 142-171, 1995. [Online] Available: http://uix.sagepub.com/content/17/2/142.reprint

[12] Expression of the Uncertainty of Measurement in Calibration, EA4/02, European cooperation for accreditation, 1999. [Online] Available: http://www.european-accreditation.org/publication/ea-402-m

[13] L. Svilainis et al., "Acquisition system for the arbitrary pulse width and position signals application in ultrasound", Sensor Letters, vol. 12, no. 9, pp. 1399-1407, 2014. [Online] Available: http://dx.doi.org/10.1166/s1.2014.3329

[14] C. E. Corcione, F. Freuli, M. Frigione, "Cold-curing structural epoxy resins: analysis of the curing reaction as a function of curing time and thickness", Materials, vol. 7, pp. 6832-6842, 2014. [Online] Available: http://www.mdpi.com/1996-1944/7/9/6832 